\title{
Mediterranean Diet Score and prostate cancer risk in a Swedish population-based case-control study
}

\author{
Elisabeth Möller ${ }^{1}$, Carlotta Galeone ${ }^{2,3}$, Therese M.-L. Andersson ${ }^{1}$, Rino Bellocco ${ }^{1,4}$, Hans-Olov Adami ${ }^{1,5}$, \\ Ove Andrén ${ }^{6}$, Henrik Grönberg ${ }^{1}$, Carlo La Vecchia ${ }^{2,3}$, Lorelei A. Mucci ${ }^{5,7}$ and Katarina Bälter ${ }^{1}$ \\ ${ }^{1}$ Department of Medical Epidemiology and Biostatistics, Karolinska Institutet, PO Box 281, Stockholm SE-171 77, Sweden \\ ${ }^{2}$ Department of Epidemiology, Mario Negri Institute for Pharmacological Research, Milan, Italy \\ ${ }^{3}$ Department of Clinical Sciences and Community Health, University of Milan, Milan, Italy \\ ${ }^{4}$ Department of Statistics and Quantitative Methods, University of Milano-Bicocca, Milan, Italy \\ ${ }^{5}$ Department of Epidemiology, Harvard School of Public Health, Boston, MA, USA \\ ${ }^{6}$ Department of Urology, Örebro University Hospital, Örebro, Sweden \\ ${ }^{7}$ Department of Medicine, Channing Laboratory, Brigham and Women's Hospital and Harvard Medical School, Boston, MA, USA
}

(Received 14 March 2012 - Final revision received 4 December 2012 - Accepted 19 December 2012)

Journal of Nutritional Science (2013), vol. 2, e15, page 1 of 13

doi:10.1017/jns.2013.2

Abstract

Several individual components of the Mediterranean diet have been shown to offer protection against prostate cancer. The present study is the first to investigate the association between adherence to the Mediterranean diet and the relative risk of prostate cancer. We also explored the usefulness of the Mediterranean Diet Score (MDS) in a non-Mediterranean population. FFQ data were obtained from 1482 incident prostate cancer patients and 1108 population-based controls in the Cancer of the Prostate in Sweden (CAPS) study. We defined five MDS variants with different components or using either study-specific intakes or intakes in a Greek reference population as cut-off values between low and high intake of each component. Unconditional logistic regression was used to estimate the relative risk of prostate cancer for high and medium $v$. low MDS, as well as potential associations with the individual score components. No statistically significant association was found between adherence to the Mediterranean diet based on any of the MDS variants and prostate cancer risk (OR range: 0.96-1.19 for total prostate cancer, comparing high with low adherence). Overall, we found little support for an association between the Mediterranean diet and prostate cancer in this Northern European study population. Despite potential limitations inherent in the study or in the build-up of a dietary score, we suggest that the original MDS with study-specific median intakes as cut-off values between low and high intake is useful in assessing the adherence to the Mediterranean diet in non-Mediterranean populations.

Key words: Prostatic neoplasms: Dietary patterns: Dietary score: Epidemiology

Prostate cancer is the second most common cancer among men globally and is the most common cancer in high-income countries $^{(1)}$. However, its incidence varies considerably in different parts of the world. The highest incidence is seen in the Nordic countries, North America, Australia and New Zealand, with age-standardised rates ranging from seventy-three to 112 per 100000 person-years $^{(1)}$. In most Mediterranean countries, the incidence rates are almost half to two-thirds lower ${ }^{(1)}$, suggesting that certain aspects of the Mediterranean lifestyle may be associated with a reduced incidence of prostate cancer.

A number of epidemiological studies suggest that a Mediterranean dietary pattern may offer protection against several types of cancer ${ }^{(2,3)}$. The traditional Mediterranean diet is the dietary habit typical of the Mediterranean regions, notably

Abbreviations: CAPS, Cancer of the Prostate in Sweden; MDS, Mediterranean Diet Score; MP:S, MUFA and PUFA to SFA.

* Corresponding author: Elisabeth Möller, fax +468314975, email elisabeth.moller@ki.se

(C) The Author(s) 2013. The online version of this article is published within an Open Access environment subject to the conditions of the Creative Commons Attribution-NonCommercial-ShareAlike licence <http://creativecommons.org/licenses/by-nc-sa/2.5/>. The written permission of 
Crete and other parts of Greece, southern Italy and Spain, in the period before the 1960s. The general features of this diet are high intake of vegetables, fruits, nuts, beans, cereals and lean fish, together with high consumption of olive oil, moderate consumption of alcohol (especially wine with meals) and low amounts of milk and red meat. Several of these individual food items, or the nutrients they contain, have been associated with a reduced risk of prostate cancer ${ }^{(4)}$. Based on previous knowledge about the effect of Mediterranean dietary components, Trichopoulou et al. ${ }^{(5)}$ estimated that shifting to a traditional Mediterranean diet could prevent up to $10 \%$ of the prostate cancer cases in Western high-income countries.

Several dietary scores have been developed to assess adherence to the Mediterranean $\operatorname{diet}^{(6)}$. In 1995, Trichopoulou et al. ${ }^{(7)}$ developed the Mediterranean Diet Score (MDS) based on eight dietary components: the intake of vegetables, fruits and nuts, legumes, cereals, meat, dairy products and alcohol, as well as the ratio of MUFA to SFA. Fish was added as a ninth component in $2003^{(8)}$. The MDS and several modified versions have been used in epidemiological studies of various health outcomes, both in Mediterranean and non-Mediterranean populations ${ }^{(6,9,10)}$. An important component of the traditional Mediterranean diet is olive oil, resulting in a high ratio of MUFA to SFA. Outside the Mediterranean region olive oil is consumed less frequently, and the intake of MUFA is not only considerably lower, but mainly originates from a higher meat intake rather than from vegetable oils. In an attempt to adapt the MDS for use in non-Mediterranean populations, the fat ratio component has been modified so as to include both MUFA and PUFA in the numerator ${ }^{(11,12)}$. Moreover, the cut-off value between high and low intake for each MDS component is the median intake in the population under study. However, using the median intake in a non-Mediterranean study population may be questionable, as the intake of certain components may be much lower or higher than that in a typical Mediterranean population. Thus the score may be less able to discriminate between intake levels that are beneficial or non-beneficial to health.

In the present study, we investigated the association between the Mediterranean diet and the relative risk of prostate cancer in a Swedish population. In order to examine the potential effect of using study-specific median intakes in a non-Mediterranean population as cut-off values in the MDS, we developed several variants of the score using both intakes in the Swedish study population and in a Greek reference population ${ }^{(8)}$ as cut-off values. We also created an alternative score that more closely reflects the traditional Mediterranean diet, directed towards its most beneficial components. Furthermore, we explored the potential effect measure modification by selected covariates. To the best of our knowledge, this is the first study specifically investigating prostate cancer risk and the Mediterranean diet using a dietary score.

\section{Experimental methods}

\section{Study population}

The Cancer of the Prostate in Sweden (CAPS) study is a population-based case-control study on prostate cancer and has been described in detail previously ${ }^{(13-15)}$. Briefly, incident and histologically confirmed prostate cancer cases were identified from four of the six regional cancer registries in Sweden and were invited to take part in the study. Cases were 35-79 years old at enrolment, from January 2001 to September 2002, and lived in the central and northern parts of Sweden. For $95 \%$ of the cases, clinical data were obtained from the National Prostate Cancer Registry. Advanced cases were defined as those meeting at least one of the following criteria: tumour, nodes, metastasis stage T3/T4 or N1 or M1; Gleason score 8-10; or serum prostate-specific antigen level at diagnosis $\geq 100 \mathrm{ng} / \mathrm{ml}$. Localised cases were defined as those with T1/T2, N0, M0, Gleason score 2-6 and prostate-specific antigen $<20 \mathrm{ng} / \mathrm{ml}$. Cases with Gleason score 7 who did not meet the criteria for advanced disease were included in neither advanced nor localised cases due to the difference in aggressiveness seen for Gleason 3+4 compared with Gleason $4+3^{(16)}$. Controls were selected randomly from the Swedish Population Registry every 6 months, being frequency-matched to cases by age in 5 -year categories and region of residence. Invitations to participate were sent out to eligible controls about once a month, except July and August, to reflect the continuous enrolment of cases.

All study participants were asked to complete a baseline questionnaire. The number of cases filling in the questionnaire was $1499(79 \%)$ out of 1895 invited and the corresponding number for controls was 1130 (67\%) out of 1684 invited. The average time between diagnosis and sending out the questionnaire was 5 months. The study was performed according to the guidelines laid down in the Declaration of Helsinki and was approved by the ethics committees at Karolinska Institutet and Umeå University in Sweden. Written informed consent was obtained from all participants.

\section{Exposure assessment}

Usual dietary intake over the past year was assessed using a semi-quantitative FFQ with 106 items, including foods, beverages and alcohol, and with three additional questions on dietary fat, including a question on regular use of olive oil for cooking and/or in dressings (yes/no) ${ }^{(13,14,17,18)}$. The intake of several food items assessed with a shorter version of the FFQ has been validated against four $7 \mathrm{~d}$ weighed food records in 111 randomly sampled Swedish women, giving Spearman correlation coefficients in the range $0 \cdot 16-0 \cdot 82$ (e.g. $0 \cdot 30$ 0.37 for vegetables/tomatoes, $0.38-0.49$ for fruit/fruit juice, $0 \cdot 16-0.61$ for refined grains/whole grains/cereals, 0.44 for fish, 0.44 for dairy products, $0.37-0.60$ for meat/poultry and $0.56-0.82$ for alcoholic beverages ${ }^{(19)}$. Although the validation was performed in women, the results are likely to be similar in men ${ }^{(20,21)}$. The intake of nutrients has been validated against fourteen repeated 24-h recall interviews in 248 randomly sampled Swedish men, showing Spearman correlation coefficients in the range $0 \cdot 25-0.81$ (e.g. 0.75 for SFA, 0.66 for MUFA, 0.49 for PUFA and 0.81 for ethanol) ${ }^{(22)}$. The energy intake assessed by the FFQ was within $8 \%$ of the estimated intake from the $24 \mathrm{~h}$ recalls in the same validation study. To calculate nutrient and energy intakes, we created 
aggregated codes corresponding to 253 food and beverage items, and linked the dietary data from the questionnaire to the Swedish National Food Administration database ${ }^{(23)}$, comprising about 1500 food products.

The questionnaire also contained questions regarding known and potential lifestyle risk factors for prostate cancer, such as physical activity, tobacco use, height, weight, education, occupation, marital status, history of diabetes and family history of cancer (prostate or other). The questions on physical activity included occupational activity, walking/bicycling, exercise and household/leisure-time activity at age 15, 30, 50 and 65 years. They have been validated against two $7 \mathrm{~d}$ activity diaries in 111 men (Spearman correlation $r 0 \cdot 56)^{(24)}$. Physical activity level was calculated as metabolic equivalent by multiplying the reported time in $\mathrm{h} / \mathrm{d}$ for each activity by the assigned metabolic equivalent value, given in appendix 1 of the publication by Norman et al. ${ }^{(24)}$. Only activities of moderate or vigorous intensity (metabolic equivalent $\geq 3$ ) were used ${ }^{(25)}$. We calculated the lifetime physical activity by taking the mean value over all ages.

\section{The Mediterranean Diet Score}

We created five variants of the MDS originally developed by Trichopoulou et al. ${ }^{(7,8)}$, as listed in Table 1. The main score, denoted as MDS-gram, is based on nine components: a high ratio of MUFA and PUFA to SFA (MP:S); high vegetable intake (including tomato juice, ketchup and root vegetables, except potatoes); high intake of fruit, nuts and seeds; high legume intake; high cereal intake; high fish and seafood intake; low intake of dairy products; low intake of meat and meat products (including poultry); and moderate alcohol intake. Frequencies of intake from the questionnaire were translated into intakes in $\mathrm{g} / \mathrm{d}$ using standard portion sizes ${ }^{(26)}$. All intakes were energy-adjusted by dividing the actual food intake by the individual's total energy intake and multiplying by $10460 \mathrm{~kJ}$ (equivalent to $2500 \mathrm{kcal})^{(7)}$. A score of zero or one was assigned to each component using the median intake among the controls in $\mathrm{g} / \mathrm{d}$ as cut-off between low and high intake. For beneficial components (the first six listed previously), one point was given for intakes at or above the median and zero points otherwise. For the milk and meat components, one point was given for intakes below the median and zero points otherwise. For alcohol (ethanol), one point was given for intakes above zero and below the median intake, and zero points otherwise. A total score ranging from zero to nine points was calculated by summing up the scores of the individual components. The total score was then categorised into low (zero to three points), medium (four to five points) or high (six to nine points) adherence to the Mediterranean diet based on the approximate tertile distribution among the controls.

A total of three score variants were created based on the same components as MDS-gram, but with different cut-off values between low and high intake of each component. In MDS-serv, we used the study-specific median intakes (among the controls) expressed as servings/week instead of $\mathrm{g} / \mathrm{d}$ as cut-off values, assuming that one serving of a food item has the same impact no matter the serving size. In MDS-cent, the study-specific twenty-fifth or seventy-fifth

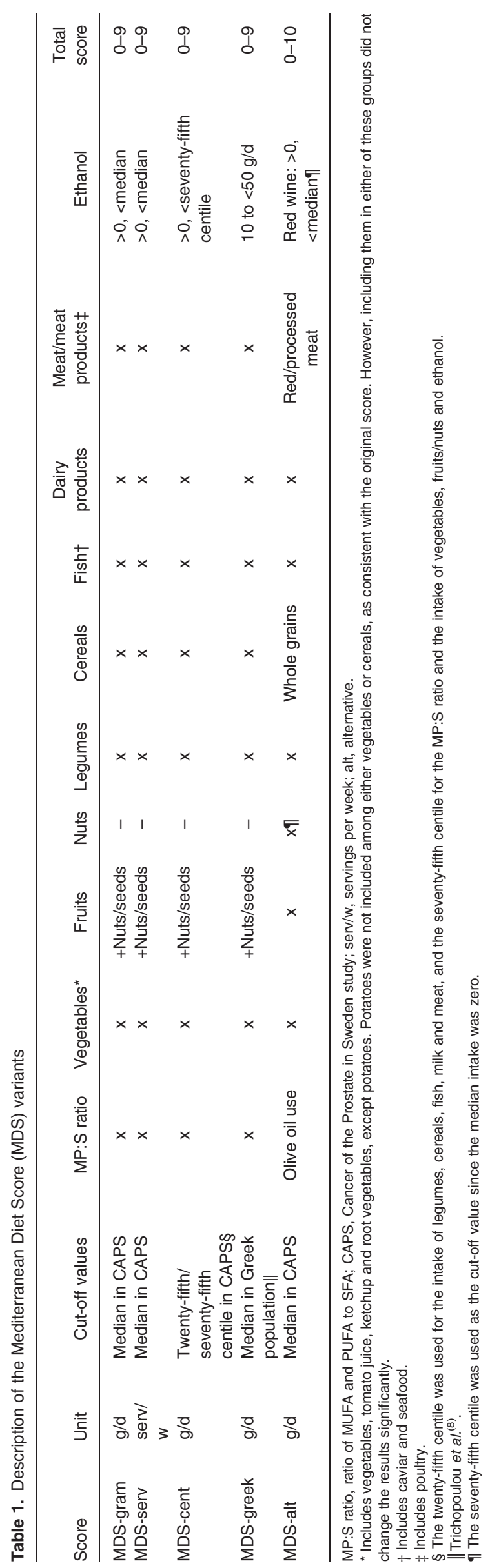


centiles of the intake (in $\mathrm{g} / \mathrm{d}$ ) were used as cut-off values instead of the median intake; the centile level for each component was chosen so as to achieve cut-off values that were close to the median intakes in the Greek reference population, while maintaining a sufficient number of participants in the extreme groups. In MDS-greek, the median intakes (in $\mathrm{g} / \mathrm{d}$ ) in the aforementioned Greek reference population were used as cut-off values; however, for vegetable and fruit/nut intake we used the ninetieth centile of the intake in the CAPS population since very few participants reached the Greek median intake levels. MDS-serv and MDS-cent were categorised using the same adherence cut-off values as MDS-gram, whereas MDSgreek was categorised as low (zero to two points), medium (three to four points), or high (five to nine points) adherence.

Lastly, we created an alternative score, denoted as MDS-alt, with a focus on the most 'traditional' components of the Mediterranean diet. This score was based on ten components, basically the same as in the other score variants except that the fat ratio was replaced by olive oil use, cereals by whole grains, meat by red/processed meat, ethanol by red wine and fruits and nuts/seeds were separated into two components. Similar variants of the MDS have been used in other studies ${ }^{(27-30)}$. Olive oil use was added as a separate component since it was not included in the nutrient calculations for fat, due to the design of the questionnaire in the CAPS study (yes/no, no frequency reported). The study-specific median intake in $\mathrm{g} / \mathrm{d}$ was used as the cut-off between low and high intake of each component. The total MDS-alt ranging from zero to ten points was categorised into low (zero to three points), medium (four to five points) or high (six to ten points) adherence to the Mediterranean diet.

\section{Statistical methods}

We excluded men with incomplete dietary data $(n 67)$ or unreasonably high or low energy intakes $(<3300 \mathrm{~kJ} / \mathrm{d}$ or $>21000$ $\mathrm{kJ} / \mathrm{d}$ ) (n 27). In total, 2590 participants (1482 cases and 1108 controls) were included in the final analyses. Baseline characteristics of cases and controls were compared using the Wilcoxon-Mann-Whitney test for continuous variables and the $\chi^{2}$ test for categorical variables. Correlations between the continuous MDS variants and their components were evaluated by estimating Spearman correlation coefficients.

Unconditional logistic regression was used to generate OR with $95 \% \mathrm{CI}$, as estimates of the relative risk of prostate cancer according to adherence to the Mediterranean diet. Adherence to each MDS variant was analysed using indicator variables for low, medium and high score, with low as the reference group. Additionally, the scores were modelled as continuous variables, firstly by assessing the linear effect of a one-point increment in the score on prostate cancer risk and secondly by fitting restricted cubic splines to relax the linear assumption and to create smoothed functions of adherence to the scores. Moreover, we evaluated the individual effects of each MDS component. All analyses were performed for total prostate cancer, for advanced ( $n$ 588) and localised ( $n$ 512) disease, as well as for Gleason score 7 disease subtype (n 218).
Simple regression models included the matching factors: age and region of residence. Multivariate regression models additionally included education, smoking, BMI, energy intake, physical activity, history of diabetes and family history of prostate cancer. Potential confounders were selected based on subject matter knowledge as well as on indications provided by purely statistical procedures, such as a change in $\beta$-coefficients $(>10 \%)$. Other covariates that were considered as potential confounders but not included in the final model were height, employment status, marital status, snuff use, use of dietary supplements, use of olive oil and intake of coffee, phyto-oestrogens, potatoes, sweet foods and non-alcoholic beverages. The potential effect measure modification by selected covariates (age, family history of prostate cancer, history of diabetes and BMI) was evaluated in two ways: formal interaction tests by including multiplicative interaction terms in logistic regression models, and by the use of interaction indicator variables to obtain a stratified effect.

Wald and likelihood ratio tests were used to assess the statistical significance of observed associations $(P<0.05$, twosided tests). The fit of the models was evaluated by the Hosmer-Lemeshow and the Pearson $\chi^{2}$ goodness-of-fit tests. All analyses were performed using the statistical software systems SAS version 9.2 (SAS Institute Inc.) and Stata version 12 (StataCorp LP).

\section{Results}

The study population is described in Table 2. The cases were somewhat younger than the controls and were more likely to reside in the northern part of Sweden, to be employed, to take dietary supplements and to have a family history of prostate cancer. Cases and controls also differed with regard to their intake of total energy, alcohol, non-alcoholic beverages, tomatoes and sweet foods, but there were no major differences concerning BMI, level of education, marital status, smoking status, physical activity, coffee intake or potato intake. The differences observed for the matching factors age and region of residence may be explained by the lower participation rate among controls than among cases, and was apparent already before exclusion of participants ( $n$ 94) (results not shown).

The mean total score of each of the five MDS variants, as well as the distribution between the three adherence groups, is given in Table 3. The results were in general similar for cases and controls, except for a slightly higher mean of MDS-serv, MDS-cent and MDS-alt among the cases. The mean total score of MDS-gram was 4.4 (SD 1.7) among both cases and controls: $31 \%$ of cases and $33 \%$ of controls had low adherence, $42 \%$ of cases and $40 \%$ of controls had medium adherence and $27 \%$ of both cases and controls had high adherence.

Table 4 presents the intake levels of the MDS components and of the total energy, macronutrients and selected food items, in the CAPS population as well as in the Greek reference population. The intake of vegetables and fruits was considerably lower in the Swedish population, whereas the intake of legumes, cereals, fish and dairy products was higher than that in the Greek population. The intake of meat was similar in both populations. As expected, the MP:S ratio in the 
Table 2. Characteristics of the cases and controls in the Cancer of the Prostate in Sweden (CAPS) study

\begin{tabular}{|c|c|c|c|c|c|c|c|}
\hline \multirow[b]{2}{*}{ Characteristics } & \multicolumn{3}{|c|}{ Cases (n 1482) } & \multicolumn{3}{|c|}{ Controls (n 1108) } & \multirow[b]{2}{*}{$P^{\star}$} \\
\hline & $n$ & & $\% †$ & $n$ & & $\% †$ & \\
\hline Age (years) & & & & & & & $<0.01$ \\
\hline Mean & & $66 \cdot 8$ & & & $67 \cdot 7$ & & \\
\hline $\mathrm{SD}$ & & $7 \cdot 3$ & & & 7.5 & & \\
\hline Region of residence & & & & & & & $<0.01$ \\
\hline Northern Sweden & 455 & & 31 & 197 & & 18 & \\
\hline Central Sweden & 1027 & & 69 & 911 & & 82 & \\
\hline Education level & & & & & & & 0.15 \\
\hline 0-9 years & 675 & & 46 & 506 & & 46 & \\
\hline 10-12 years & 595 & & 40 & 468 & & 42 & \\
\hline$\geq 13$ years & 207 & & 14 & 127 & & 11 & \\
\hline Marital status & & & & & & & 0.25 \\
\hline Married/partner & 1200 & & 81 & 867 & & 78 & \\
\hline Divorced/unmarried & 204 & & 14 & 165 & & 15 & \\
\hline Widower & 77 & & 5 & 71 & & 6 & \\
\hline History of diabetes & & & & & & & 0.62 \\
\hline Yes & 171 & & 12 & 134 & & 12 & \\
\hline No & 1292 & & 87 & 953 & & 86 & \\
\hline Family history of prostate cancer & & & & & & & $<0.01$ \\
\hline Yes & 273 & & 18 & 103 & & 9 & \\
\hline No & 1209 & & 82 & 1005 & & 91 & \\
\hline Smoking & & & & & & & 0.44 \\
\hline Never smoker & 575 & & 39 & 424 & & 38 & \\
\hline Former smoker & 734 & & 49 & 535 & & 48 & \\
\hline Current smoker & 156 & & 11 & 134 & & 12 & \\
\hline Dietary supplement use & & & & & & & $<0.01$ \\
\hline Yes & 702 & & 47 & 437 & & 39 & \\
\hline No & 737 & & 50 & 623 & & 56 & \\
\hline BMI at inclusion $\left(\mathrm{kg} / \mathrm{m}^{2}\right)$ & & & & & & & 0.44 \\
\hline$<25$ & 574 & & 39 & 400 & & 36 & \\
\hline 25 to $<30$ & 686 & & 46 & 526 & & 47 & \\
\hline$\geq 30$ & 177 & & 12 & 141 & & 13 & \\
\hline Physical activity (MET-h/d)‡ & & & & & & & 0.94 \\
\hline Mean & & $12 \cdot 7$ & & & $12 \cdot 8$ & & \\
\hline SD & & 8.9 & & & $9 \cdot 1$ & & \\
\hline Total energy intake $(\mathrm{kJ} / \mathrm{d})$ & & & & & & & $<0.01$ \\
\hline Mean & & 9583.5 & & & 9303.9 & & \\
\hline SD & & $2670 \cdot 2$ & & & $2721 \cdot 1$ & & \\
\hline Alcohol intake (g/d) & & & & & & & $<0.01$ \\
\hline Mean & & $8 \cdot 2$ & & & $8 \cdot 0$ & & \\
\hline SD & & $16 \cdot 8$ & & & $19 \cdot 9$ & & \\
\hline Non-alcoholic beverage intake (servings/d) & & & & & & & $<0.01$ \\
\hline Mean & & 1.0 & & & 0.9 & & \\
\hline SD & & 1.2 & & & $1 \cdot 2$ & & \\
\hline Coffee intake (cups/d) & & & & & & & 0.67 \\
\hline Mean & & $3 \cdot 1$ & & & $3 \cdot 1$ & & \\
\hline SD & & 1.9 & & & $2 \cdot 0$ & & \\
\hline Tomato intake (servings/d) & & & & & & & $<0.01$ \\
\hline Mean & & 0.4 & & & 0.4 & & \\
\hline SD & & 0.3 & & & 0.3 & & \\
\hline Potato intake (servings/d) & & & & & & & $0 \cdot 19$ \\
\hline Mean & & 0.5 & & & 0.5 & & \\
\hline SD & & 0.3 & & & 0.3 & & \\
\hline Sweet foods intake (servings/d)§ & & & & & & & $<0.01$ \\
\hline Mean & & 0.8 & & & 0.8 & & \\
\hline SD & & 0.5 & & & 0.5 & & \\
\hline Disease characteristics among cases ( $\%$ of all cases) & & & & & & & - \\
\hline Advanced\| & 588 & & 40 & - & & - & \\
\hline Localisedๆ & 512 & & 34 & - & & - & \\
\hline Gleason score 7 & 218 & & 15 & - & & - & \\
\hline Unknown & 87 & & 6 & - & & - & \\
\hline Other & 77 & & 5 & - & & - & \\
\hline Gleason score & & & & & & & - \\
\hline Mean & & 6.5 & & & - & & \\
\hline SD & & 1.2 & & & - & & \\
\hline
\end{tabular}


Table 2. Continued

\begin{tabular}{|c|c|c|c|c|c|c|}
\hline \multirow[b]{2}{*}{ Characteristics } & \multicolumn{2}{|c|}{ Cases ( $n$ 1482) } & \multicolumn{3}{|c|}{ Controls ( $n$ 1108) } & \multirow[b]{2}{*}{$P^{*}$} \\
\hline & $n$ & $\% \dagger$ & $n$ & & $\% \dagger$ & \\
\hline PSA level (ng/ml) & & & & & & - \\
\hline Mean & & & & - & & \\
\hline SD & & & & - & & \\
\hline
\end{tabular}

MET, metabolic equivalent; PSA, prostate-specific antigen.

* Two-sided $P$ values estimated using the $\chi^{2}$ test for categorical variables and the Wilcoxon-Mann-Whitney test for continuous variables.

† Percentage of all cases and of all controls, respectively. Column percentages do not always add up to 100 due to missing data.

$\ddagger$ Activity of at least moderate intensity (MET $\geq 3$ ).

$\S$ Includes confectionery, sweet bakery products and ice cream.

|| Meeting at least one of the following criteria: tumour stage T3/T4 or N1 or M1; or Gleason score 8-10; or PSA $\geq 100 \mathrm{ng} / \mathrm{ml}$.

I) Meeting all of the following criteria: tumour stage T1/T2, NO, MO, Gleason score 2-6 and PSA $<20 \mathrm{ng} / \mathrm{ml}$.

Swedish population was lower than the MUFA:SFA ratio in the Greek population. The intake of SFA was similar in both populations, whereas the intake of MUFA and PUFA, as well as the total energy, was lower in the Swedish population. The Swedish population ate more potatoes and sweet

Table 3. Adherence to the Mediterranean Diet Score (MDS) variants in cases and controls in the Cancer of the Prostate in Sweden (CAPS) study* (Mean values and standard deviations; number of participants and percentages)

\begin{tabular}{|c|c|c|c|c|}
\hline \multirow[b]{2}{*}{ Characteristics } & \multicolumn{2}{|c|}{$\begin{array}{c}\text { Cases } \\
(n 1482)\end{array}$} & \multicolumn{2}{|c|}{$\begin{array}{l}\text { Controls } \\
(n 1108)\end{array}$} \\
\hline & $n$ & $\% \dagger$ & $n$ & $\% \dagger$ \\
\hline \multicolumn{5}{|l|}{ MDS-gram (points) } \\
\hline Mean & \multicolumn{2}{|c|}{4.4} & \multicolumn{2}{|c|}{4.4} \\
\hline SD & \multicolumn{2}{|c|}{$1 \cdot 7$} & \multicolumn{2}{|c|}{$1 \cdot 7$} \\
\hline \multicolumn{5}{|l|}{ MDS-serv (points) } \\
\hline Mean & \multicolumn{2}{|c|}{4.4} & \multicolumn{2}{|c|}{$4 \cdot 3$} \\
\hline SD & \multicolumn{2}{|c|}{1.7} & \multicolumn{2}{|c|}{1.7} \\
\hline \multicolumn{5}{|l|}{ MDS-cent (points) } \\
\hline Mean & \multicolumn{2}{|c|}{$4 \cdot 2$} & \multicolumn{2}{|c|}{$4 \cdot 1$} \\
\hline SD & \multicolumn{2}{|c|}{1.5} & \multicolumn{2}{|c|}{1.5} \\
\hline \multicolumn{5}{|l|}{ MDS-greek (points) } \\
\hline Mean & \multicolumn{2}{|c|}{$3 \cdot 3$} & \multicolumn{2}{|c|}{$3 \cdot 3$} \\
\hline SD & \multicolumn{2}{|c|}{1.2} & \multicolumn{2}{|c|}{$1 \cdot 1$} \\
\hline \multicolumn{5}{|l|}{ MDS-alt (points) } \\
\hline Mean & \multicolumn{2}{|c|}{4.6} & \multicolumn{2}{|c|}{4.5} \\
\hline SD & \multicolumn{2}{|c|}{1.9} & \multicolumn{2}{|c|}{1.9} \\
\hline Adherence to MDS-gram & & & & \\
\hline Low (0-3 points) & 460 & 31 & 360 & 33 \\
\hline Medium (4-5 points) & 621 & 42 & 445 & 40 \\
\hline High (6-9 points) & 401 & 27 & 303 & 27 \\
\hline Adherence to MDS-serv & & & & \\
\hline Low (0-3 points) & 482 & 33 & 359 & 32 \\
\hline Medium (4-5 points) & 600 & 40 & 456 & 41 \\
\hline High (6-9 points) & 400 & 27 & 293 & 27 \\
\hline Adherence to MDS-cent & & & & \\
\hline Low (0-3 points) & 493 & 33 & 387 & 35 \\
\hline Medium (4-5 points) & 723 & 49 & 517 & 47 \\
\hline High (6-9 points) & 266 & 18 & 204 & 18 \\
\hline Adherence to MDS-greek & & & & \\
\hline Low (0-2 points) & 339 & 23 & 262 & 24 \\
\hline Medium (3-4 points) & 939 & 63 & 703 & 63 \\
\hline High (5-9 points) & 204 & 14 & 143 & 13 \\
\hline Adherence to MDS-alt & & & & \\
\hline Low (0-3 points) & 449 & 30 & 367 & 33 \\
\hline Medium (4-5 points) & 524 & 36 & 405 & 37 \\
\hline High (6-10 points) & 509 & 34 & 336 & 30 \\
\hline
\end{tabular}

* For details of the MDS variants, see Table 1.

† Percentage of all cases and of all controls, respectively. foods but drank less non-alcoholic beverages than the Greek population. The correlation between individual components and the main score, MDS-gram, was low to moderate, ranging from $r_{s} 0.07$ for ethanol to $r_{s} 0.58$ for vegetables. Similarly, the individual components of the alternative score, MDS-alt, were weakly or moderately correlated with the total score (range $r_{s}$ 0.05-0.46). Inter-correlation between the individual components was in the range $r_{s} 0 \cdot 02-0 \cdot 40$ (results not shown). The correlation coefficients between the MDS variants ranged from 0.43 between MDS-serv and MDS-greek, to 0.79 between MDS-gram and MDS-serv and between MDS-gram and MDS-alt, respectively (results not shown).

As shown in Fig. 1, no statistically significant association was found between any of the MDS variants and prostate cancer. However, high MDS-cent, MDS-greek and MDS-alt scores seemed to be associated with a 20-35\% increased relative risk of advanced prostate cancer, compared with low scores, and a somewhat weaker increased risk of total prostate cancer. However, the estimates were not statistically significant and the CI were wide. For cases with Gleason score 7, a nonstatistically significant inverse association was seen for high $v$. low MDS-gram and MDS-serv (multivariate OR were $0 \cdot 84$, $95 \%$ CI 0.59, 1.19; and 0.77, $95 \%$ CI 0.55, 1.09, respectively; results not shown). No association was seen between Gleason score 7 disease subtype and any of the other MDS-variants. There was no evidence of an association between the continuous scores and prostate cancer, in neither logistic regression models nor restricted cubic spline regression models, except for a non-significant positive association between the total prostate cancer and MDS-cent (results not shown).

Among the individual score components, a high vegetable intake was associated with an increased risk of prostate cancer, with multivariate OR $1.25\left(95 \%\right.$ CI $\left.1.05,1.50 ; P_{\text {trend }}=0.01\right)$ for total prostate cancer, $1.37\left(95 \%\right.$ CI 1.09, 1.72; $P_{\text {trend }}=$ 0.006 ) for advanced disease and 1.25 (95\% CI 0.98, 1.60; $P_{\text {trend }}=0.07$ ) for localised disease (results not shown). In supplementary analyses, the positive association remained but was weakened when tomatoes were excluded from vegetable intake, and a high intake of tomato products was found to be positively associated with localised disease (multivariate OR 1.25; $95 \%$ CI 0.99, 1.59; $P_{\text {trend }}=0.07$ ) compared with low intake. A high ethanol intake was associated with an increased risk of advanced prostate cancer (multivariate OR 1.25; $95 \%$ CI 0.99, 1.58; $P_{\text {trend }}=0.06$ ). Simple models 


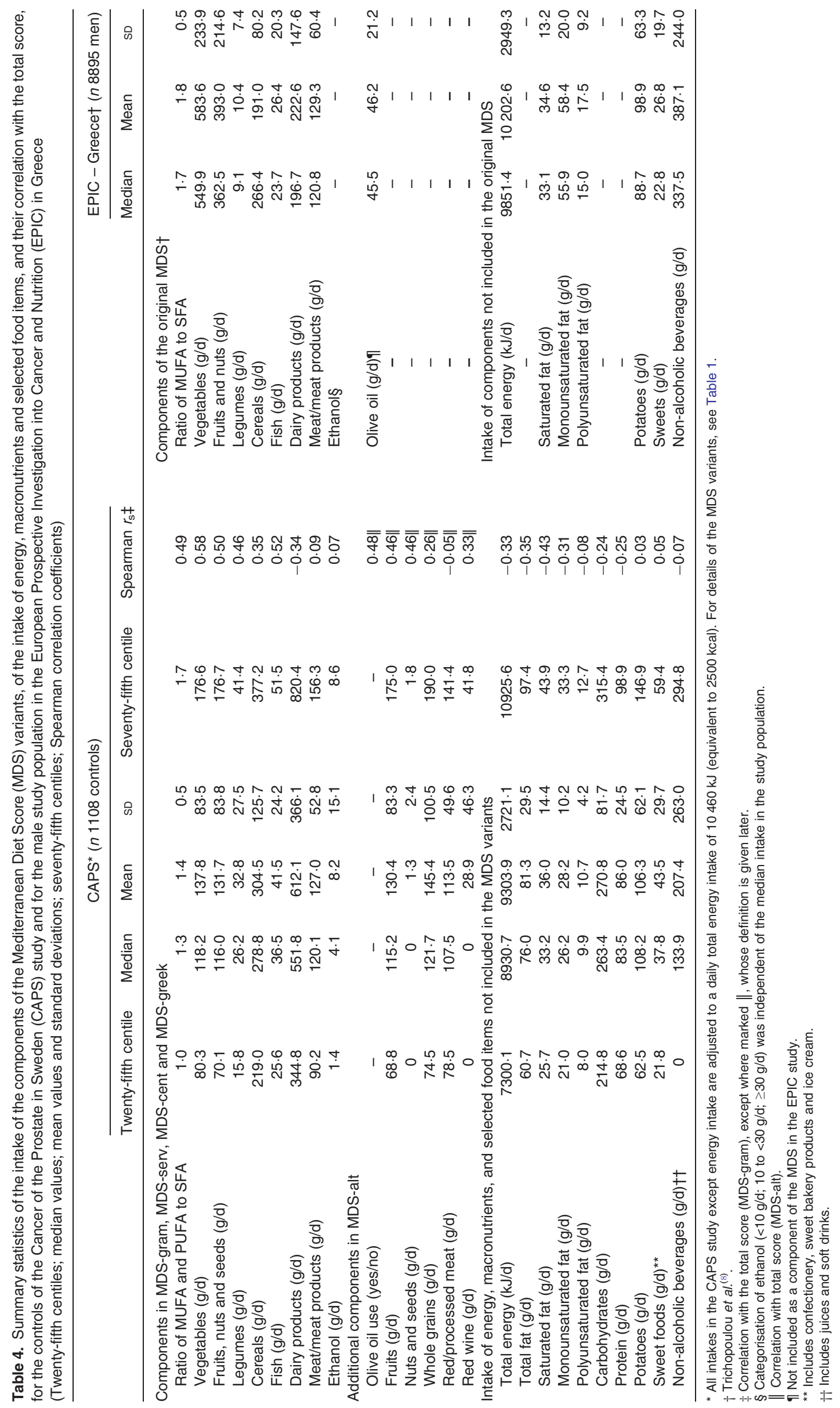


(a)

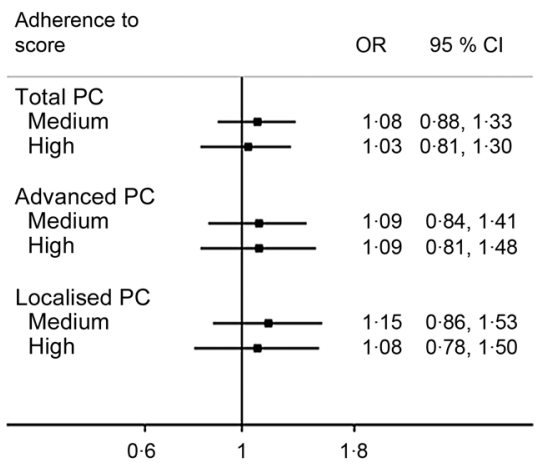

(b)

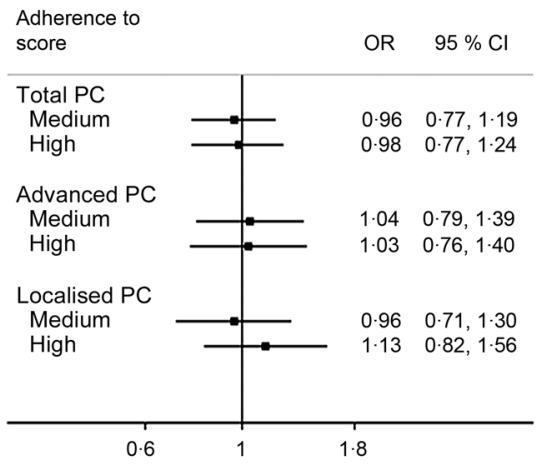

(c)

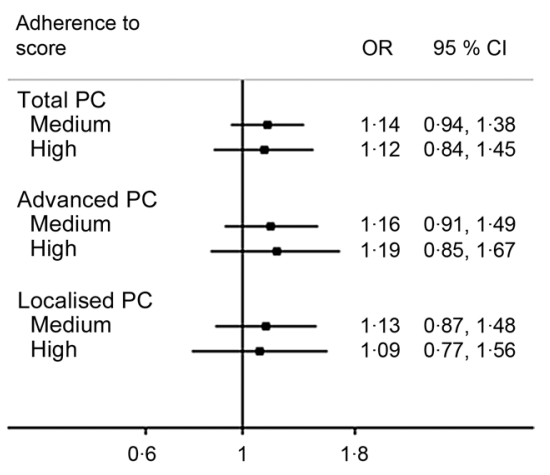

(d)

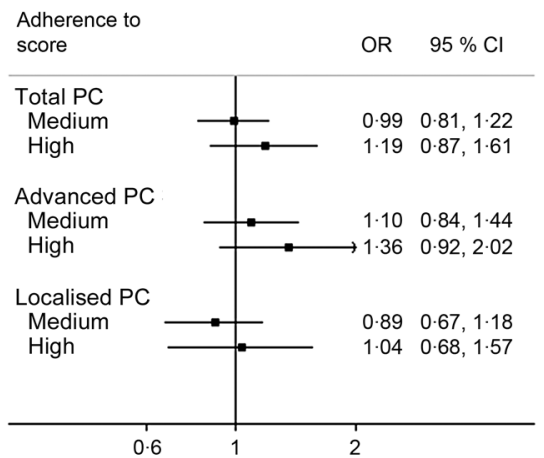

(e)

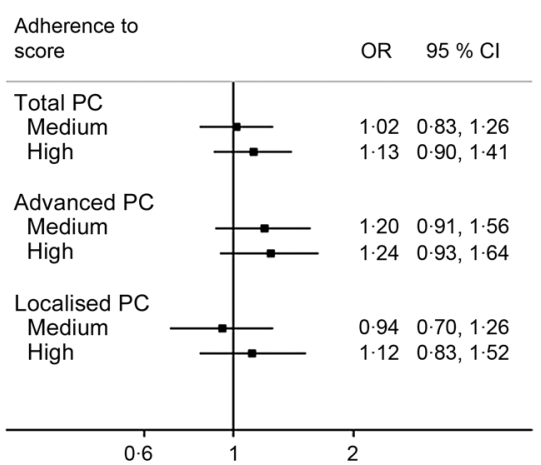

Fig. 1. Relative risk of total ( $n$ 2336), advanced ( $n$ 1494) and localised ( $n$ 1441) prostate cancer (PC) according to adherence to the Mediterranean Diet Score (MDS) variants: (a) MDS-gram, (b) MDS-serv, (c) MDS-cent, (d) MDS-greek and (e) MDS-alt. For details of the MDS variants, see Table 1 . Estimates reflect OR (95 \% CI) derived from unconditional logistic regression models. All models are adjusted for age (in 5-year intervals), region of residence (north/central), education (0-9 years; 10-12 years; $\geq 13$ years), smoking status (never/former/current), BMl (quartile distribution of controls), energy intake (quartile distribution of controls), physical activity (quartile distribution of controls), history of diabetes (yes/no) and family history of prostate cancer (yes/no). Adherence groups were categorised as follows: (a-c) low adherence, 0-3 points (reference); medium adherence, 4-5 points; high adherence, 6-9 points; (d) low adherence, 0-2 points (reference); medium adherence, 3-4 points; high adherence, 5-9 points; (e) low adherence, 0-3 points (reference); medium adherence, 4-5 points; high adherence, 6-10 points.

showed an inverse association for a high MP:S ratio, but the association disappeared after adjusting for potential confounders. A high meat intake was associated with a 10-15\% reduction in prostate cancer risk in multivariate models, although this was not statistically significant. No association was found between the intake of fruits and nuts, legumes, cereals, fish or dairy products and prostate cancer. The results for Gleason score 7 disease subtype were in the same direction as for total prostate cancer (results not shown). As illustrated in Fig. 2, smoothed functions of intake showed weak positive associations between total prostate cancer and the MP:S ratio, vegetable intake and fruit/nut intake, although the CI included the value of 1 . A dose-response trend was suggested for the association with the MP:S ratio. A high intake of legumes, fish and meat was possibly associated with a non-significant reduced risk of prostate cancer, whereas no association was observed for cereals, dairy products or alcohol.

Analysis of components specific to the MDS-alt showed an inverse association between a high intake of red/processed meat and total prostate cancer (multivariate OR 0.83; $95 \%$ CI $\left.0.70,0.99 ; P_{\text {trend }}=0.04\right)$. The results were similar but nonstatistically significant in the case of advanced and localised disease. We observed an increased risk of advanced disease for a high intake of red wine (multivariate OR 1.39; $95 \%$ CI $1.06,1 \cdot 82 ; P_{\text {trend }}=0 \cdot 02$ ). No statistically significant associations were seen for olive oil use, whole grains, fruit or nuts.
Tests for potential interactions between the Mediterranean diet (MDS-gram) and selected covariates showed no statistically significant interactions (results not shown). However, when data were stratified using interaction indicator variables, men with a family history of prostate cancer had a non-significant increased risk of total prostate cancer (OR 1.67; $95 \%$ CI 0.87; 3.22; $\left.P_{\text {interaction }}=0.29\right)$ comparing high with low MDS-gram, which was not seen in men without a family history of the disease; this possible interaction was even more pronounced for advanced disease (OR 2.42; $95 \%$ CI 1.10, 5.34; $\left.P_{\text {interaction }}=0 \cdot 10\right)$. Furthermore, stratified analyses showed a positive association between the Mediterranean diet and prostate cancer among obese men $\left(\mathrm{BMI} \geq 30 \mathrm{~kg} / \mathrm{m}^{2}\right)$, with multivariate OR $2 \cdot 06(95 \%$ CI 1.11, 3.81; $\left.P_{\text {interaction }}=0.09\right)$ for total malignancy, $2 \cdot 17(95 \%$ CI $0.99,4.76 ; P_{\text {interaction }}=0.24$ ) for advanced disease and 1.94 (95\% CI 0.84, 4.49; $\left.P_{\text {interaction }}=0.48\right)$ for localised disease, but not among men with BMI $<30 \mathrm{~kg} / \mathrm{m}^{2}$. Age at inclusion and diabetes did not act as effect modifiers in the present study.

\section{Discussion}

Overall, we found no association between the Mediterranean diet and relative risk of prostate cancer. Among individual score components, a high intake of vegetables and alcohol was associated with an increased risk of prostate cancer, and a high intake of red/processed meat with a decreased risk of 
(a)

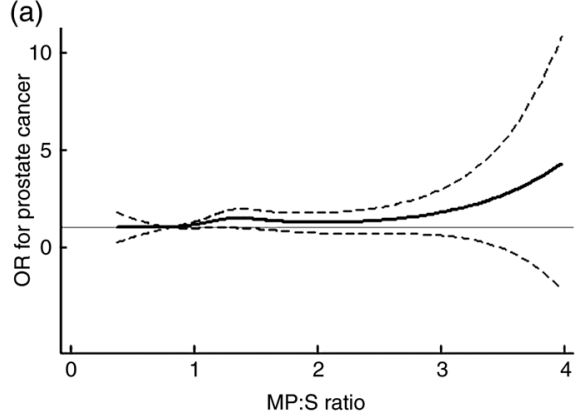

(d)

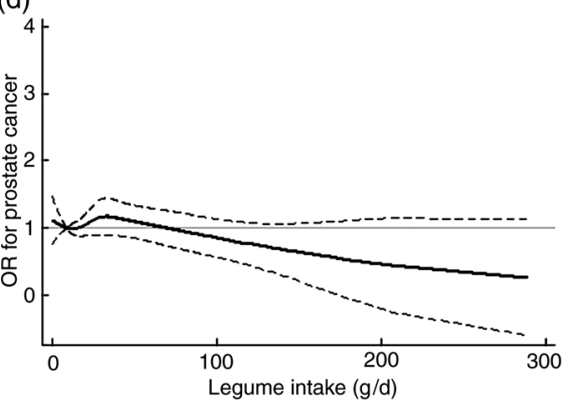

(g)

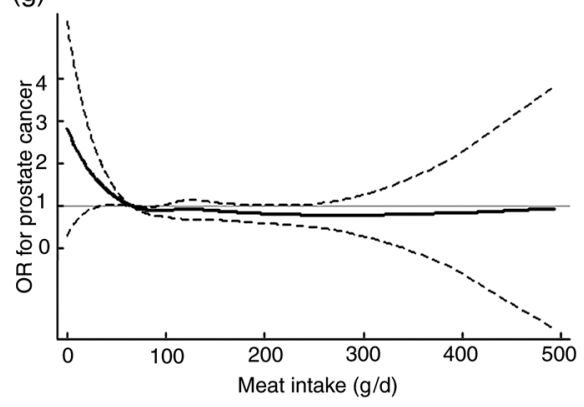

(b)

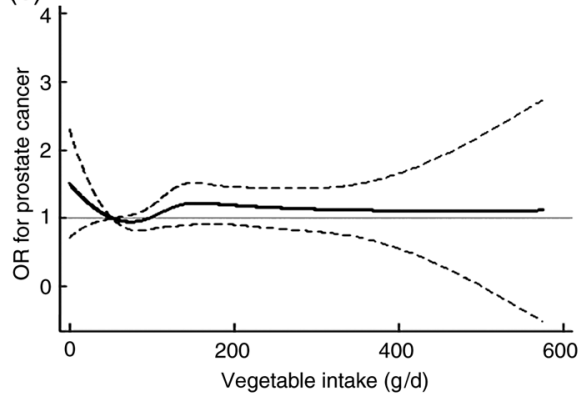

(e)

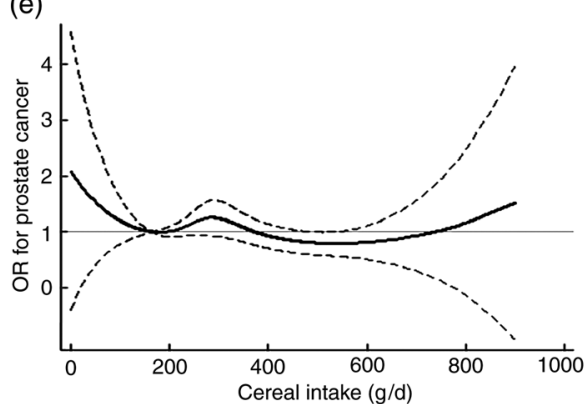

(h)

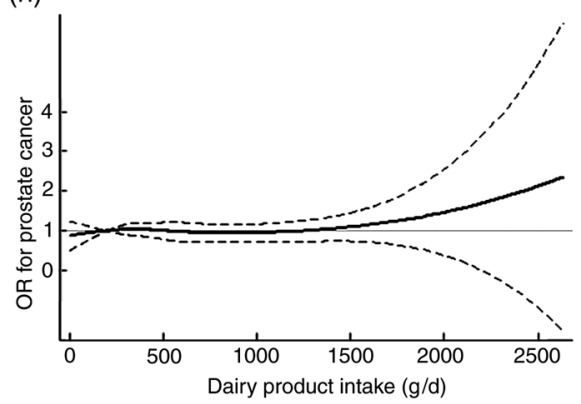

(c)

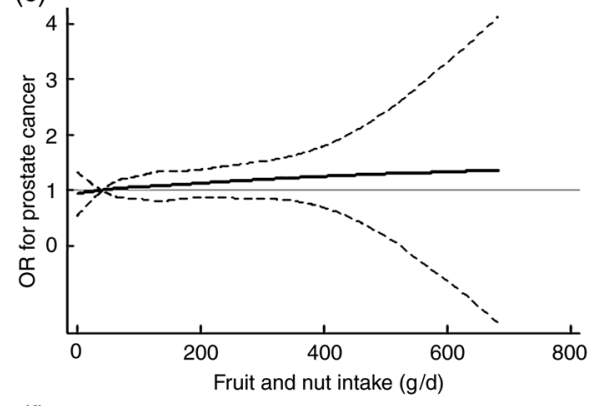

(f)

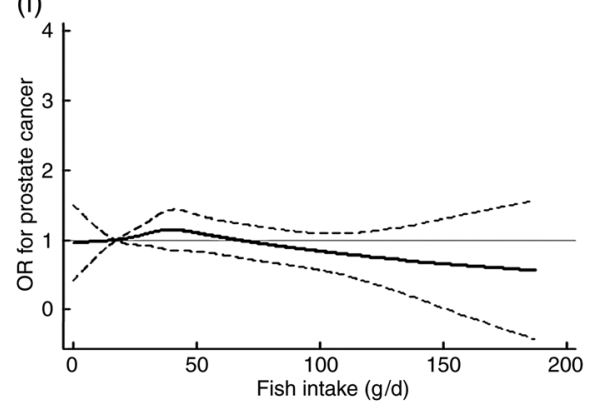

(i)

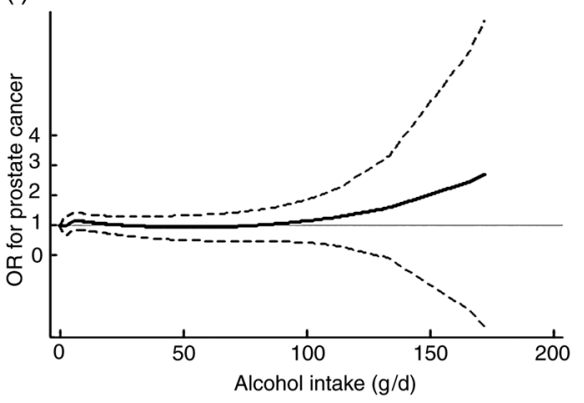

Fig. 2. Dose-response relationship between components of the Mediterranean Diet Score (MDS-gram; see Table 1 for details) and relative risk of total prostate cancer ( $n$ 2336): (a) MUFA and PUFA to SFA (MP:S) ratio, (b) vegetables, (c) fruits and nuts, (d) legumes, (e) cereals, (f) fish, (g) meat and meat products, (h) dairy products and (i) alcohol. Results from restricted cubic regression splines with five knots. (-), OR; (----), $95 \% \mathrm{Cl}$. The Cl cross at the reference point, equivalent to the tenth centile of the intake in the study population. Regression models include age (in 5-year intervals), region of residence (north/central), education (0-9 years; 10-12 years; $\geq 13$ years), smoking status (never/former/current), BMI (quartile distribution of controls), energy intake (quartile distribution of controls), physical activity (quartile distribution of controls), history of diabetes (yes/no) and family history of prostate cancer (yes/no).

the disease. The association between MDS and prostate cancer was indicated as positive in obese men and in men with a family history of the disease.

To the best of our knowledge, no other studies have so far been carried out on the association between the Mediterranean dietary pattern and prostate cancer using a dietary score. A few studies looking at empirically derived dietary patterns have shown an increased risk of prostate cancer with 'Western' dietary patterns rich in meat and processed foods ${ }^{(31-33)}$, i.e. foods consumed at low amounts in the traditional Mediterranean diet. Interestingly, Greek migrants in Australia have a lower risk of prostate cancer than men born in Australia, and they have also retained the dietary habits of their native country, as opposed to many other migrant populations ${ }^{(4)}$. Studies on the Mediterranean diet and overall cancer risk have shown inconsistent results ${ }^{(2,3)}$. A meta-analysis of data from seven cohort studies provided a pooled relative risk of cancer of $0.94(95 \%$ CI $0.92,0.96)$ for a two-point increase in the $\operatorname{MDS}^{(9)}$. In a recent review, the Mediterranean diet was considered as 'probably' protective against overall cancer, albeit there is variation with regard to different cancer sites ${ }^{(3)}$.
Although the Mediterranean diet as a whole has not previously been studied in relation to prostate cancer, its individual components have. Recent reviews suggest that a high intake of vegetables, marine fatty acids, legumes and foods containing vitamin $\mathrm{E}$ (such as vegetable oils, nuts and seeds) may offer protection against the disease ${ }^{(4,34-37)}$, and a favourable effect may also be seen with a low intake of meat and foods rich in $\mathrm{Ca}$ such as dairy products ${ }^{(4,35-38)}$. In theory, the combined effect of these components may be beneficial in prostate cancer prevention.

In the present study, the observed positive association between a high vegetable intake and prostate cancer is unexpected, considering earlier indications of an inverse association $^{(34,36)}$. The main contributors to the vegetable intake were tomatoes, carrots and mixed vegetables, each contributing on average $14-16 \%$. A high intake of tomatoes, notably tomato sauce, has been shown in several studies to reduce the risk of prostate cancer ${ }^{(34,37,39)}$. The indicated positive association between tomato intake and localised disease in our study may be explained by localised cases potentially being more health conscious and more likely to eat healthier. 
The increased risk for the total vegetable intake was strongest for advanced disease and remained, although weakened, when tomatoes were excluded from the vegetable intake. Also, the vegetable intake was much lower in the study population than in the Greek reference population, and thus a true detrimental effect is unlikely. We also observed positive associations between advanced disease and intakes of total alcohol and red wine, although there is limited evidence for an association between alcohol and prostate cancer ${ }^{(36,37,40)}$. Moreover, our findings of an inverse association with red/processed meat and no association with dairy products are in contrast with previous findings of an increased risk of prostate cancer for high intakes of meat and $\mathrm{Ca}$, although the evidence is inconsistent ${ }^{(36,37,41,42)}$. The deviating results in our study may be explained by residual confounding or recall bias, or may be due to chance, considering that we tested multiple hypotheses. Another component of the MDS that deserves attention is the fish intake. Previous studies on the intake of fatty fish rich in $n$-3 fatty acids have shown inverse associations with prostate cancer ${ }^{(43,44)}$, whereas a recent meta-analysis of the total fish intake showed no association ${ }^{(45)}$. The MDS does not distinguish between fatty and non-fatty fish, which may explain the lack of association in our study.

Olive oil is the main source of fat in the Mediterranean region and was included as a component of the alternative score, MDS-alt. It has been shown to protect against several types of cancer ${ }^{(46,47)}$, but no consistent association has been found with prostate cancer ${ }^{(48)}$. We observed no association between olive oil use and prostate cancer. Due to the generally low intake of olive oil in the Swedish population at the time of data collection, this component probably has little overall influence on the present results.

The construction of a dietary score as a measure of a healthy diet is complex, and the MDS is no exception. An inappropriate scoring method may be unable to detect a true association or may lead to misclassification of exposure. For instance, the score may be too blunt to detect a weak association with cancer since it does not capture all dietary aspects or factors unrelated to diet, or because the beneficial and non-beneficial components of the score may counter-balance each other's potential effects, leading to a null result. This is a possible explanation for the overall lack of association seen in the present study. Also, strong correlations between the score and its components may influence the discriminatory power of each individual component. In the present study, the estimated inter-correlation between all components and their correlation with the total score was low to moderate, which means that each component contributes equally to the score. An exception was the vegetable component with a relatively high correlation with the total score $\left(r_{s} 0.58\right)$; hence, it may be the dominant factor determining the score. Milà-Villarroel $e t a l .{ }^{(49)}$ recently evaluated the reliability of several indexes assessing adherence to the Mediterranean diet, and the MDS showed high performance.

Using study-specific intakes, as opposed to externally defined values, as cut-off between low and high intake increases the statistical power in each intake group, but it does not necessarily mean that the score is able to discriminate between beneficial and non-beneficial health effects. This may be problematic especially in non-Mediterranean populations where intake levels may be very different from those of a typical Mediterranean population. The main differences between our Swedish study population and the Greek reference population were the considerably lower intake of fruit and vegetables and higher dairy product intake in the former. However, the two populations differ not only in nationality but also in age, the CAPS population being older, and in the type of exposure data, the Greek data being longitudinal and prospective whereas the Swedish data were collected only once and retrospectively. Nevertheless, the observed differences in intake could indicate a limited discriminatory power of the MDS in the study population of the present study.

To explore the influence of using study-specific intakes as cut-off values, we compared variants of the MDS with different cut-off values, including one score based on the median intake in a Greek reference population. Overall, we did not find any statistically significant association with prostate cancer for any of the score variants. The non-significant increased risk of disease found with MDS-cent and MDS-greek was unexpected, as we had rather anticipated inverse associations. These results are probably driven by a high vegetable intake, as the vegetable component had the highest correlation with the total score and was positively associated with prostate cancer risk, especially advanced disease. Since overall we observed no major differences between the score variants, we suggest that the use of study-specific intakes as cut-off values in the MDS is appropriate also in non-Mediterranean populations. The usefulness of the MDS in non-Mediterranean populations is further supported by an Australian study where the MDS was found to be appropriate for the prediction of survival in both a population of Mediterranean descent (Greek-Australians) and a non-Mediterranean population (Anglo-Celts) ${ }^{(50)}$. Also, in a European multi-centre cohort study, a two-point increment in the MDS yielded risk estimates for overall cancer that were similar in men in Northern and Southern European countries ${ }^{(51)}$.

We also created an alternative score variant, MDS-alt, to investigate the effect of a more specific score that better reflects the 'original' Mediterranean diet. Replacing the fat ratio with olive oil use, cereals with whole grains, meat with red/processed meat and ethanol with red wine, as well as separating fruits from nuts, ideally captures the most health-beneficial components of the Mediterranean diet. Comparing MDS-gram and MDS-alt, which both used study-specific median intakes as cut-off values, we found no statistically significant associations for either of them, the two scores being highly correlated. The nonstatistically significantly increased relative risk of advanced prostate cancer seen with MDS-alt is surprising, and may be driven by the vegetable component as previously discussed, or may be due to residual confounding.

The influence of diet on risk of developing prostate cancer may differ between men who are diagnosed in young or old age, or between men with and without a family history of the disease, since the type of prostate cancer and disease mechanisms may differ between the groups. We also hypothesised that diabetic men, as well as overweight or obese men, may have different metabolic responses to a Mediterranean diet compared with non-diabetic or normal-weight men. Formal 
interaction tests showed no statistically significant interactions for any of these factors. However, in stratified analyses we observed positive associations between adherence to the Mediterranean diet and prostate cancer among men with a family history of prostate cancer and among obese men, but not among men without a family history of the disease or who were non-obese. These results should be interpreted with caution, especially since the control groups were relatively small ( $n 89$ for those with a family history of prostate cancer and $n 129$ for $\mathrm{BMI} \geq 30 \mathrm{~kg} / \mathrm{m}^{2}$ ).

The strengths of our study include the large sample size, high response-rate, the use of population-based random controls, complete and rapid ascertainment of prostate cancer cases and clinical information on prostate cancer subtypes. The proportion of prostate-specific antigen-detected cases was low (29\% of 1499$)$ due to the low frequency of prostatespecific antigen testing at the time of enrolment ${ }^{(14)}$, ensuring a high proportion of cases with clinically relevant prostate cancer. The risk of confounding by population stratification was minimised by the ethnic homogeneity of the study population. Also, the extensive questionnaire provided detailed information on both exposure and potential confounding factors.

Some limitations with the case-control design need to be considered in the interpretation of the present results. Selection bias is possible since cases are generally more prone to participate than controls, and selection of healthconscious controls may occur. The retrospective collection of exposure information may produce recall bias. However, a previous study comparing original and repeated dietary recall interviews showed very little or no overall difference in performance between prostate cancer cases and non-cases ${ }^{(52)}$. Another drawback is the fact that the FFQ assesses dietary intake 1 year prior to diagnosis, which may differ from dietary habits earlier in life, before tumour initiation. Furthermore, reverse causation bias cannot be ruled out, although men $>60$ years generally tend to maintain their dietary habits even after a cancer diagnosis ${ }^{(53-55)}$.

Due to limitations in the method for exposure assessment, measurement error is likely to occur. FFQ generally underestimate total energy intake as a result of their inability to capture the whole diet. The mean and median energy intakes in the CAPS study population were considerably lower than 10 $600 \mathrm{~kJ}$, the recommended daily energy intake for sedentary men $61-74$ years of age ${ }^{(56)}$, which could indicate an underestimation of total food intake. Since energy intake was significantly different between cases and controls, differential misclassification of exposure is possible. FFQ may also overestimate the usual intake of those food items actually asked for, leading to distorted estimates of intake ${ }^{(57)}$, and social desirability may lead to over-reporting of healthy foods and under-reporting of unhealthy foods. The validation study of an FFQ almost identical to the one used in the CAPS study showed low to moderate correlation for several food items $^{(19)}$; however, high correlation coefficients between FFQ and food records are rarely achieved ${ }^{(58)}$. Another study validating a modified MDS version assessed by an FFQ against 24-h recalls showed a Pearson's correlation coefficient of $0 \cdot 48$, and the average FFQ-based score was $102 \%$ of the recall-based score ${ }^{(59)}$. In the present study, the use of energy-adjusted dietary intake reduces the potential influence of bias due to measurement error. Besides, such errors have less influence on the ranking of individuals, which is our main objective, compared with assessment of absolute intake ${ }^{(57)}$. The potential effect of any remaining measurement error on our relative risk estimates is attenuation, which would reduce the likelihood of detecting a potentially weak diet-disease relationship ${ }^{(57)}$; thus it may in part explain the lack of an association seen between the Mediterranean diet and prostate cancer.

In conclusion, we found no support for any association between the Mediterranean dietary pattern and prostate cancer in the Swedish CAPS study. We have compared several MDS variants with different compositions and cut-off values, and observed no significant associations with any of the scores. We suggest that using study-specific median intakes as cut-off values as in the original MDS is an appropriate way of assessing adherence to the Mediterranean diet also in non-Mediterranean populations. However, the present results, including the unexpected associations seen for certain components of the MDS, need to be cautiously interpreted in light of potential limitations in the case-control design, the exposure assessment or the discriminatory power of the MDS in our study population. Further studies are needed to clarify the relationship between the Mediterranean diet and prostate cancer risk.

\section{Acknowledgements}

The present study was funded by the Swedish Cancer Society (grant no. 08 0470). The work of R. B. was partially supported by the Italian Ministry of Education and Research (MIUR, PRIN 2009 X8YCBN). The work of C. G. and C. L. V. was supported by the Italian Association for Research on Cancer (AIRC, grant no. 10068). The authors declare no conflicts of interest. We would like to acknowledge Vassiliki Benetou for valuable feedback regarding the MDS, and all those involved in the CAPS study, notably the participants, the urologists and data collectors, and the Regional Cancer Registries. The authors' contributions were as follows: E. M. and K. B. planned the study; E. M. analysed the data and wrote the manuscript; C. G., R. B., T. M. A., C. L. V. and L. A. M. participated in the planning of the study and the analysis and interpretation of the data; O. A., H. G., H.-O. A. and $\mathrm{K}$. B. were involved in designing and conducting the CAPS study; and K. B. obtained the funding of and supervised the present study. All authors have taken part in critical revision of the manuscript and have approved the final version. This material was presented in part at the Eighth International Conference on Diet and Activity Methods, 14-17 May 2012, Rome, Italy; the Eleventh European Nutrition Conference, 26-29 October 2011, Madrid, Spain; and was published (in part) in: the Eleventh European Nutrition Conference (FENS). Ann Nutr Metab (2011) 58, Suppl. 3, 273-274.

\section{References}

1. Ferlay J, Shin HR, Bray F, et al. (2010) GLOBOCAN 2008 Cancer Incidence and Mortality Worldwide: Version 1.2. IARC CancerBase 
No. 10. Lyon, France: International Agency for Research on Cancer. http://globocan.iarc.fr (accessed August 2012).

2. Kontou N, Psaltopoulou T, Panagiotakos D, et al. (2011) The Mediterranean diet in cancer prevention: a review. J Med Food 14, 1065-1078.

3. Verberne L, Bach-Faig A, Buckland G, et al. (2010) Association between the Mediterranean diet and cancer risk: a review of observational studies. Nutr Cancer 62, 860-870.

4. Itsiopoulos C, Hodge A \& Kaimakamis M (2009) Can the Mediterranean diet prevent prostate cancer? Mol Nutr Food Res 53, 227-239.

5. Trichopoulou A, Lagiou P, Kuper H, et al. (2000) Cancer and Mediterranean dietary traditions. Cancer Epidemiol Biomarkers Prev 9, 869-873.

6. Bach A, Serra-Majem L, Carrasco JL, et al. (2006) The use of indexes evaluating the adherence to the Mediterranean diet in epidemiological studies: a review. Public Health Nutr 9, 132-146.

7. Trichopoulou A, Kouris-Blazos A, Wahlqvist ML, et al. (1995) Diet and overall survival in elderly people. BMJ 311, 1457-1460.

8. Trichopoulou A, Costacou T, Bamia C, et al. (2003) Adherence to a Mediterranean diet and survival in a Greek population. $N$ Engl J Med 348, 2599-2608.

9. Sofi F, Abbate R, Gensini GF, et al. (2010) Accruing evidence on benefits of adherence to the Mediterranean diet on health: an updated systematic review and meta-analysis. Am J Clin Nutr 92, 1189-1196.

10. Waijers PM, Feskens EJ \& Ocke MC (2007) A critical review of predefined diet quality scores. Br J Nutr 97, 219-231.

11. Knoops KT, de Groot LC, Kromhout D, et al. (2004) Mediterranean diet, lifestyle factors, and 10-year mortality in elderly European men and women: the HALE project. JAMA 292, 1433-1439.

12. Trichopoulou A, Orfanos P, Norat T, et al. (2005) Modified Mediterranean diet and survival: EPIC-elderly prospective cohort study. BMJ 330, 991.

13. Chang ET, Hedelin M, Adami HO, et al. (2005) Alcohol drinking and risk of localized versus advanced and sporadic versus familial prostate cancer in Sweden. Cancer Causes Control 16, 275-284.

14. Hedelin M, Klint A, Chang ET, et al. (2006) Dietary phytoestrogen, serum enterolactone and risk of prostate cancer: the cancer prostate Sweden study (Sweden). Cancer Causes Control 17, 169-180.

15. Lindmark F, Zheng SL, Wiklund F, et al. (2004) H6D polymorphism in macrophage-inhibitory cytokine- 1 gene associated with prostate cancer. J Natl Cancer Inst 96, 1248-1254.

16. Stark JR, Perner S, Stampfer MJ, et al. (2009) Gleason score and lethal prostate cancer: does $3+4=4+3$ ? J Clin Oncol 27, 3459-3464.

17. Chang ET, Smedby KE, Zhang SM, et al. (2005) Dietary factors and risk of non-Hodgkin lymphoma in men and women. Cancer Epidemiol Biomarkers Prev 14, 512-520.

18. Westerlund A, Steineck G, Balter K, et al. (2011) Dietary supplement use patterns in men with prostate cancer: the Cancer Prostate Sweden study. Ann Oncol 22, 967-972.

19. Khani BR, Ye W, Terry P, et al. (2004) Reproducibility and validity of major dietary patterns among Swedish women assessed with a food-frequency questionnaire. J Nutr 134, 1541-1545.

20. Sasaki S, Kobayashi M \& Tsugane S (2003) Validity of a selfadministered food frequency questionnaire used in the 5-year follow-up survey of the JPHC Study Cohort I: comparison with dietary records for food groups. J Epidemiol 13, Suppl. 1, S57-S63.

21. Toft U, Kristoffersen L, Ladelund S, et al. (2008) Relative validity of a food frequency questionnaire used in the Inter99 study. Eur J Clin Nutr 62, 1038-1046.

22. Messerer M, Johansson SE \& Wolk A (2004) The validity of questionnaire-based micronutrient intake estimates is increased by including dietary supplement use in Swedish men. J Nutr 134, 1800-1805.

23. Bergström L, Kylberg E, Hagman U, et al. (1991) The food composition database KOST: the National Food Administration's Information System for nutritive values of food. V årFöda 43, 439-447.
24. Norman A, Bellocco R, Bergstrom A, et al. (2001) Validity and reproducibility of self-reported total physical activity-differences by relative weight. Int J Obesity 25, 682-688.

25. Ainsworth BE, Haskell WL, Herrmann SD, et al. (2011) 2011 Compendium of Physical Activities: a second update of codes and MET values. Med Sci Sports Exerc 43, 1575-1581.

26. Livsmedelsverket (2001) Vikttabell, version 01.2. Uppsala, Sweden: National Food Agency.

27. Gonzalez CA, Argilaga S, Agudo A, et al. (2002) Sociodemographic differences in adherence to the Mediterranean dietary pattern in Spanish populations. Gac Sanit 16, 214-221.

28. Schroder H, Marrugat J, Vila J, et al. (2004) Adherence to the traditional Mediterranean diet is inversely associated with body mass index and obesity in a Spanish population. J Nutr 134, 3355-3361.

29. Fung TT, Hu FB, McCullough ML, et al. (2006) Diet quality is associated with the risk of estrogen receptor-negative breast cancer in postmenopausal women. J Nutr 136, 466-472.

30. Nkondjock A \& Ghadirian P (2007) Diet quality and BRCA-associated breast cancer risk. Breast Cancer Res Treat 103, 361-369.

31. Ambrosini GL, Fritschi L, de Klerk NH, et al. (2008) Dietary patterns identified using factor analysis and prostate cancer risk: a case control study in Western Australia. Ann Epidemiol 18, 364-370.

32. De Stefani E, Ronco AL, Deneo-Pellegrini H, et al. (2010) Dietary patterns and risk of advanced prostate cancer: a principal component analysis in Uruguay. Cancer Causes Control 21, 1009-1016.

33. Walker M, Aronson KJ, King W, et al. (2005) Dietary patterns and risk of prostate cancer in Ontario, Canada. Int J Cancer 116, 592-598.

34. Chan R, Lok K \& Woo J (2009) Prostate cancer and vegetable consumption. Mol Nutr Food Res 53, 201-216.

35. Hori S, Butler E \& McLoughlin J (2011) Prostate cancer and diet: food for thought? BJU Int 107, 1348-1359.

36. Ma RW \& Chapman K (2009) A systematic review of the effect of diet in prostate cancer prevention and treatment. J Hum Nutr Diet 22, 187-199; quiz 200-202.

37. World Cancer Research Fund/American Institute for Cancer Research (2007) Food, Nutrition, Physical Activity and the Prevention of Cancer: A Global Perspective. Washington, DC: AICR.

38. Niclis C, Diaz Mdel P, Eynard AR, et al. (2012) Dietary habits and prostate cancer prevention: a review of observational studies by focusing on South America. Nutr Cancer 64, 23-33.

39. Wei MY \& Giovannucci EL (2012) Lycopene, tomato products, and prostate cancer incidence: a review and reassessment in the PSA screening era. J Oncol 2012, 271063.

40. Rota M, Scotti L, Turati F, et al. (2012) Alcohol consumption and prostate cancer risk: a meta-analysis of the dose-risk relation. Eur J Cancer Prev 21, 350-359.

41. Alexander DD, Mink PJ, Cushing CA, et al. (2010) A review and meta-analysis of prospective studies of red and processed meat intake and prostate cancer. Nutr J 9, 50.

42. Huncharek M, Muscat J \& Kupelnick B (2008) Dairy products, dietary calcium and vitamin $\mathrm{D}$ intake as risk factors for prostate cancer: a meta-analysis of 26769 cases from 45 observational studies. Nutr Cancer 60, 421-441.

43. Augustsson K, Michaud DS, Rimm EB, et al. (2003) A prospective study of intake of fish and marine fatty acids and prostate cancer. Cancer Epidemiol Biomarkers Prev 12, 64-67.

44. Hedelin M, Chang ET, Wiklund F, et al. (2007) Association of frequent consumption of fatty fish with prostate cancer risk is modified by COX-2 polymorphism. Int J Cancer 120, 398-405.

45. Szymanski KM, Wheeler DC \& Mucci LA (2010) Fish consumption and prostate cancer risk: a review and meta-analysis. Am J Clin Nutr 92, 1223-1233.

46. Escrich E, Solanas M, Moral R, et al. (2006) Are the olive oil and other dietary lipids related to cancer? Experimental evidence. Clin Transl Oncol 8, 868-883.

47. Pelucchi C, Bosetti C, Negri E, et al. (2011) Olive oil and cancer risk: an update of epidemiological findings through 2010. Curr Pharm Des 17, 805-812. 
48. Psaltopoulou T, Kosti RI, Haidopoulos D, et al. (2011) Olive oil intake is inversely related to cancer prevalence: a systematic review and a meta-analysis of 13800 patients and 23340 controls in 19 observational studies. Lipids Health Dis 10, 127.

49. Mila-Villarroel R, Bach-Faig A, Puig J, et al. (2011) Comparison and evaluation of the reliability of indexes of adherence to the Mediterranean diet. Public Health Nutr 14, 2338-2345.

50. Kouris-Blazos A, Gnardellis C, Wahlqvist ML, et al. (1999) Are the advantages of the Mediterranean diet transferable to other populations? A cohort study in Melbourne, Australia. Br J Nutr 82, 57-61.

51. Couto E, Boffetta P, Lagiou P, et al. (2011) Mediterranean dietary pattern and cancer risk in the EPIC cohort. Br J Cancer 104, 1493-1499.

52. Wilkens LR, Hankin JH, Yoshizawa CN, et al. (1992) Comparison of long-term dietary recall between cancer cases and noncases. $\mathrm{Am} \mathrm{J}$ Epidemiol 136, 825-835.

53. Demark-Wahnefried W, Peterson B, McBride C, et al. (2000) Current health behaviors and readiness to pursue life-style changes among men and women diagnosed with early stage prostate and breast carcinomas. Cancer 88, 674-684.

54. Mroz LW, Chapman GE, Oliffe JL, et al. (2010) Men, food, and prostate cancer: gender influences on men's diets. Am J Mens Health 5, 177-187.

55. Patterson RE, Neuhouser ML, Hedderson MM, et al. (2003) Changes in diet, physical activity, and supplement use among adults diagnosed with cancer. J Am Diet Assoc 103, 323-328.

56. Nordic Council of Ministers (2004) Nordic Nutrition Recommendations 2004: Integrating Nutrition and Physical Activity, 4th ed. Copenhagen, Denmark: Nordic Council of Ministers.

57. Willett W (1998) Nutritional Epidemiology, 2nd ed. New York: Oxford University Press.

58. Willett WC (1994) Future directions in the development of food-frequency questionnaires. Am J Clin Nutr 59, Suppl. 1, S171-S174.

59. Benitez-Arciniega AA, Mendez MA, Baena-Diez JM, et al. (2011) Concurrent and construct validity of Mediterranean diet scores as assessed by an FFQ. Public Health Nutr 14, 2015-2021. 